PHYSICAL REVIEW D, VOLUME 61, 019902(E)

\title{
Erratum: Proof of factorization for diffractive hard scattering [Phys. Rev. D 57, 3051 (1998)]
}

John C. Collins

(Published 29 November 1999)

PACS number(s): 13.85.Ni, 12.38.Aw, 13.60.-r, 99.10.+g

Figures 1 and 5 should be replaced by the ones below. The original figures showed the final state associated with the proton as consisting purely of the diffractive proton $p^{\prime}$. In fact, as shown in the replacement figures, there are other particles in this part of the final state; these form what is often termed the "Pomeron beam jet."

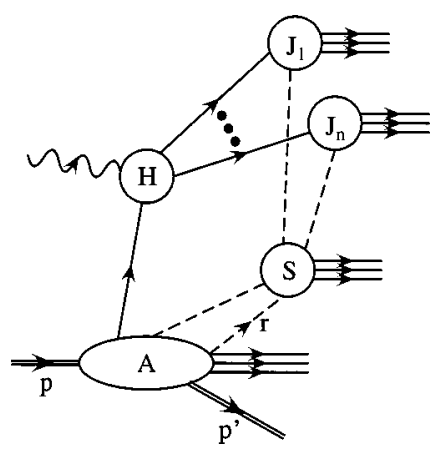

FIG. 1. Leading regions for diffrative DIS.

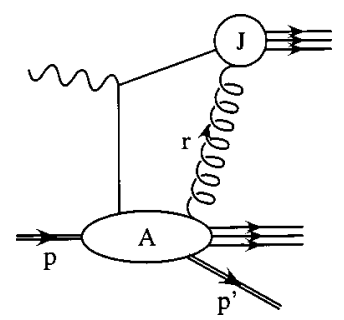

FIG. 5. Soft gluon exchange between proton subgraph and jet subgraph. 